\title{
An investigation of nodal structures and the construction of trial wave functions
}

\author{
Dario Bressanini, ${ }^{\text {a) }}$ Gabriele Morosi, ${ }^{\text {b) }}$ and Silvia Tarasco ${ }^{\text {c) }}$ \\ Dipartimento di Scienze Chimiche ed Ambientali, Universita' dell'Insubria, Sede di Como, via Lucini 3, \\ 22100 Como, Italy
}

(Received 17 August 2005; accepted 3 October 2005; published online 23 November 2005)

\begin{abstract}
The factors influencing the quality of the nodal surfaces, namely, the atomic basis set, the single-particle orbitals, and the configurations included in the wave-function expansion, are examined for a few atomic and molecular systems. The following empirical rules are found: the atomic basis set must be fairly large, complete active space and natural orbitals are usually better than Hartree-Fock orbitals, multiconfiguration expansions perform better than single-determinant wave functions, but only few configurations are effective and their choice is suggested by symmetry considerations, while too long determinantal expansions spoil the nodal surfaces. These rules allow us to reduce the nodal error and to compute the best fixed node-diffusion Monte Carlo energies for a series of dimers of first-row atoms. (C) 2005 American Institute of Physics.
\end{abstract}

[DOI: $10.1063 / 1.2128672]$

\section{INTRODUCTION}

The antisymmetric character of the electronic wave function is efficiently introduced in the diffusion Monte Carlo (DMC) method through the fixed-node approximation (FN-DMC). ${ }^{1}$ It is well known that one could exactly simulate the system of interest if only the exact nodes were available. Several exact simulation schemes that do not rely on the knowledge of the exact nodes have been proposed, ${ }^{2-7}$ but none of them, at the current state of the development, appears to be able to cope with more than a handful of electrons. The fixed-node approximation introduces the so-called nodal error, and the calculated energy in general is only an upper bound to the exact energy. Past experiences showed that the FN-DMC method is able to usually recover more than $90 \%$ of the correlation energy for small systems, performing better than standard $a b$ initio methods, but this is not enough to get chemical accuracy $(1 \mathrm{kcal} / \mathrm{mole}$ $=0.0016$ hartree $/$ molecule). Only the cancellation of the nodal error when computing dissociation energies ${ }^{8}$ or electron affinities ${ }^{9}$ has allowed obtaining of very good results. The main problem is that the fixed-node approximation is uncontrolled, and it is not possible to know in advance if a certain wave function has good nodes. Even worse, there is not yet a way to systematically generate wave functions with better nodes for a given system. The problem of reducing the nodal error to reach chemical accuracy on a sound basis has been tackled by several authors, ${ }^{8,10-15}$ studying the effect either of the atomic basis set or of the single-particle orbitals [Hartree-Fock (HF), complete active space (CAS), natural orbitals (NOs), and density-functional theory (DFT)], or of the length of the determinantal expansion. Flad et al. ${ }^{15}$ were the first to systematically investigate the seemingly paradoxi-

\footnotetext{
${ }^{a)}$ Electronic mail: dario.bressanini@uninsubria.it

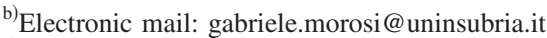

${ }^{\text {c) }}$ Electronic mail: silvia.tarasco@uninsubria.it
}

cal effect that having a wave function with a better variational energy does not necessarily lead to better fixed-node energies. It is possible that while the variational energy improves as more configuration state functions (CSFs) are added to the expansion, the FN-DMC energy gets worse. In that seminal paper, the authors concluded that it is necessary to get a better understanding of how CSFs influence the nodes.

In this paper we try to examine, on several small systems, the effect of different factors on the quality of the nodes, namely, the atomic basis set, the generation of the single-particle orbitals, and the configuration state functions added to the multideterminant expansion.

The general form of the wave function in our analysis is a determinantal part times a Jastrow correlation factor which, being positive everywhere, does not modify the location of the node.

\section{Be ATOM}

The first system we examined is the beryllium atom, a very well-known case where the nodes of a singledeterminant wave function are analytically defined by the equation

$$
\left(r_{1}-r_{2}\right)\left(r_{3}-r_{4}\right)=0,
$$

where 1 and 2 being $\alpha$ electrons and 3 and $4 \beta$ electrons. In this case the choice of the atomic basis set and of the singleparticle orbitals does not influence the FN-DMC energy. The HF nodes were shown to have the wrong topology: ${ }^{16}$ they divide the ground-state trial function into four nodal regions, while it has been proved that the exact ground-state wave function must have exactly two nodal volumes: one where the wave function is positive and the other where it is negative.

For this reason a single-determinant wave function is not sufficient to obtain the exact energy, and other configurations 
must be added. It is interesting to note that simply adding more terms to the determinant expansion does not necessarily improve the FN-DMC energy. Consider, for example, the expansion $1 s^{2} 2 s^{2}+1 s^{2} n s m s$. This trial wave function has exactly the same nodes as the HF wave function, that is, $\left(r_{1}-r_{2}\right)\left(r_{3}-r_{4}\right)=0$. The reason is that both configurations have the same algebraic structure $\left|f_{1}\left(r_{1}\right) f_{2}\left(r_{2}\right)\right|\left|f_{3}\left(r_{3}\right) f_{4}\left(r_{4}\right)\right|$. Any linear combination of configurations built only with $s$ orbitals will necessarily lead to a trial wave function with exactly the HF nodes. Of course the variational energy improves, but the fixed-node energy will remain the same. In the worst case the added configurations might even introduce some spurious nodes and increase the fixed-node energy.

To change the nodes and have a topologically correct nodal surface it is necessary to include the configuration $1 s^{2} 2 p^{2}$. Several FN-DMC calculations are reported in the literature using a trial wave function with the two configurations $1 s^{2} 2 s^{2}+1 s^{2} 2 p^{2}$. Apart from the earlier value of -14.6672(2) hartree by Lüchow and Anderson, ${ }^{8}$ which has an error bar one order of magnitude larger than the more recent results of $-14.66719(3),{ }^{17}-14.66726(1),{ }^{18}$ and $-14.66726(1)$ hartree, ${ }^{10}$ no value has the exact energy of -14.6673555 hartree ${ }^{19}$ within one error bar, although with four determinants it is possible to get $99.9 \%$ of the correlation energy. We confirmed this with an independent calculation, employing a fairly large basis set: $2(1 s) 3(2 s) 2(3 s)$ $3(2 p) 1(3 d) .^{20}$ Using two CSFs $\left(1 s^{2} 2 s^{2}+1 s^{2} 2 p^{2}\right)$ we get $-14.66729(2)$ hartree, a slightly better energy that those previously reported in the literature. This strongly suggests that convergence with respect to the atomic basis set has not been achieved yet, so we performed calculations with increasingly larger basis sets, starting from the atomic Hartree-Fock basis set by Clementi and Roetti ${ }^{21}$ plus a $2 p$ orbital and adding more $2 s$ orbitals. An extra $2 s$ orbital improves the nodal surface of the four-determinant wave function enough to get a FN-DMC energy of -14.667 34(4) hartree that has the exact value within one error bar. To investigate whether the effect of a smaller atomic-orbital basis set can be compensated by a longer configuration-interaction (CI) expansion, using the original Clementi and Roetti basis set and two $2 p$ orbitals, we added more $1 s^{2} n p m p$ configurations, but the nodes failed to improve. Although it is not possible to obtain the analytical form of the nodes of the $1 s^{2} n p m p$ in an explicit closed form, unlike the $1 s^{2} n s m s$ case, we observe the same behavior: adding more configurations of the same kind does not improve the nodes. It is interesting to note that all configurations of the kind $1 s^{2} n p_{x} m p_{x}$, for example, have the same algebraic structure $\left|f_{1}\left(r_{1}\right) x_{2} f_{2}\left(r_{2}\right)\right|\left|f_{3}\left(r_{3}\right) x_{4} f_{4}\left(r_{4}\right)\right|$.

To check the conjecture that higher angular momentum orbitals might be needed to improve the energy, we added the $1 s^{2} 3 d^{2}$ configuration. We observed a small improvement at all time steps employed, but the extrapolated value was statistically indistinguishable with the energy obtained with the $1 s^{2} 2 s^{2}+1 s^{2} 2 p^{2}$ wave function.

It would be interesting to understand why certain configurations contribute to the convergence towards the exact node and others do not. Only a speculation is possible at this point, but it is interesting to observe that in this case the useful configurations discovered so far $\left(1 s^{2} 2 s^{2}\right.$ and $\left.1 s^{2} 2 p^{2}\right)$ belong to higher and different symmetry groups than the exact wave function. They all have $S$ symmetry, being rotationally invariant, but are eigenfunctions corresponding to different eigenvalues if one considers operators other than the rotation. For example, the $1 s^{2} 2 s^{2}$ is invariant with respect to the inversion of two electrons of the same spin, while the $1 s^{2} 2 p^{2}$ configuration changes its sign. Another way to see this is to consider that the overlap integral between these configurations is zero, and they interact through the potential term, which is only rotationally invariant. A possibility is that the symmetry of the nodes of Be is higher than the symmetry of the wave function itself. This phenomenon has been observed in other atomic cases ${ }^{22}$ and in a molecular case (see $\mathrm{He}_{2}^{+}$below).

\section{THE $\mathrm{He}_{2}^{+}$SYSTEM}

\section{The exact eigenfunction: Symmetry requirements}

The Be atom does not allow to investigate the influence on the nodal structure of the atomic-orbital basis set and of the single-particle orbitals with a simple single-determinant wave function. So we choose as second system the $\mathrm{He}_{2}^{+}$ion. This three-electron system is a good, realistic, and nontrivial model to study the properties of the many-body nodal surfaces in a molecular environment.

Its ground-state has ${ }^{2} \Sigma_{u}^{+}$symmetry and the wave function is an object defined in a nine-dimensional space

$$
\Psi(\mathbf{R})=\Psi(1,2,3)=\Psi\left(x_{1}, y_{1}, z_{1}, x_{2}, y_{2}, z_{2}, x_{3}, y_{3}, z_{3}\right) .
$$

Without losing generality, we set the origin of the coordinate system in the middle of the bond, and align the molecule along the $z$ axis. A different coordinate system could be constructed by specifying, for each electron, the distances from the two nuclei, $r_{A}$ and $r_{B}$, and the azimuthal angle $\varphi$, i.e., the angle between the plane including the $z$ axis and the electron and the $y z$ plane. Using this coordinate system we can write the wave function as

$$
\Psi(\mathbf{R})=\Psi\left(r_{1 A}, r_{1 B}, \varphi_{1}, r_{2 A}, r_{2 B}, \varphi_{2}, r_{3 A}, r_{3 B}, \varphi_{3}\right) .
$$

However, since a $\Sigma$ wave function has cylindrical symmetry, it is possible to factor out a global azimuthal angle and use only eight variables to describe the ground state,

$$
\Psi(\mathbf{R})=\Psi\left(r_{1 A}, r_{1 B}, r_{2 A}, r_{2 B}, r_{3 A}, r_{3 B}, \varphi_{12}, \varphi_{13}\right),
$$

where $\varphi_{12}=\varphi_{1}-\varphi_{2}$ and $\varphi_{13}=\varphi_{1}-\varphi_{3}$. This is only one of the many possible coordinate systems, and it is not necessary, at this point, to specify the coordinate system for which such a factorization is performed.

For this molecule it is not possible to factorize the wave function into a product of a spin part and a space part. However, as long as we are only interested in spin-independent properties, we can arbitrarily assign spin to electrons. For example, we can assign spin $\alpha$ to electrons 1 and 3, and spin $\beta$ to electron 2 . In order to satisfy the Pauli principle, the function must be antisymmetric with respect to the exchange of electrons 1 and 3, thus

$$
\Psi(1,2,3)=-\Psi(3,2,1),
$$




$$
\Psi(1,2,3)=f(1,2,3)-f(3,2,1),
$$

where $f(1,2,3)$ is a generic function. However, this choice is not sufficient to completely specify the spin state, since such a wave function would have both doublet and quartet components. It can be shown ${ }^{23}$ that the following constraint provides a necessary and sufficient condition for having a pure doublet state satisfying the Pauli principle:

$$
\Psi(1,2,3)+\Psi(3,1,2)+\Psi(2,3,1)=0 .
$$

An alternative way to generate a wave function with the proper symmetry is to start with a generic function $f(1,2,3)$ multiplied by a pure doublet spin eigenfunction, for example, $[\alpha(1) \beta(2)-\beta(1) \alpha(2)] \alpha(3)$, and to apply the antisymmetrizer operator. To project the resulting spin-space wave function against a particular spin product, for example, $\alpha(1) \beta(2) \alpha(3)$, amounts to assigning the specified spin labels to the electrons. With the above choice, the most generic wave function that can be written with the correct permutational symmetry is

$$
\Psi(\mathbf{R})=f(1,2,3)+f(2,1,3)-f(2,3,1)-f(3,2,1) .
$$

\section{The nodal surface: Symmetry requirements}

The nodal surface is implicitly defined by the equation $\Psi(\mathbf{R})=0$ and so it is an eight-dimensional object. However, a consequence of the dimensional reduction is that only seven variables are sufficient to describe the nodal surface. When we say that the node is a seven-dimensional (7D) surface, we mean that to uniquely identify any point on this surface we must specify, in general, seven coordinates, the eighth being given implicitly by the node-defining equation. In general, the dimensionality of a surface embedded in $\mathfrak{R}^{N}$ space is $N-M$, where $M$ is the number of constraints (equations) that the surface must satisfy. Note that, topologically, only an $(N-1)$-dimensional surface is able to divide an $N$-dimensional region. This does not mean that the nodal surface cannot be described by fewer variables than $N-1$. It only means that the shape of the node is "flat" (constant) in the remaining coordinates.

Any symmetry constraint imposed on the wave function is automatically imposed also on its nodes. For example, suppose we fix the positions of the three electrons and rotate them jointly around the $z$ axis. Since the wave function has cylindrical symmetry, its value remains constant as we rotate the electrons. This remains true if we choose the electron positions, such that the wave function is zero.

In one-dimensional systems the nodes of the wave functions can sometimes be fixed by symmetry. For example, the node of the first excited state of the harmonic oscillator can be inferred by symmetry to be exactly at the origin, without knowing the wave function. Unfortunately symmetry constraints are usually not sufficient to completely specify the nodes for many-body systems. The Pauli principle implies that the spatial wave function must be zero when two electrons with the same spin are at the same point, i.e., $\mathbf{r}_{i}=\mathbf{r}_{j} \Rightarrow \Psi(\mathbf{R})=0$. This is equivalent to the three constraints $x_{i}=x_{j}, y_{i}=y_{j}$, and $z_{i}=z_{j}$. This set of points, sometimes called the "Pauli hyperplane," has dimension $N-3$ and does not completely specify the full $(N-1)$-dimensional node. It is only a sort of "scaffolding" through which the rest of the node must pass. A common misconception is that $\Psi(\mathbf{R})=0$ only when two electrons with the same spin are at the same position. The above argument shows that this is not true, and the wave function can be zero even when two electrons are at an arbitrary distance.

Taking into account the cylindrical symmetry of the state, other subsets of the full nodal surfaces can be specified. For example, the wave function is zero if $r_{1 A}=r_{3 A}, r_{1 B}=r_{3 B}$, and $\varphi_{12}=\varphi_{23}$. However, this is still an $(N-3)$-dimensional set.

\section{The restricted Hartree-Fock (RHF) wave function: Symmetry requirements}

The restricted Hartree-Fock ground-state wave function for $\mathrm{He}_{2}^{+}$is

$$
\Psi_{\mathrm{RHF}}(\mathbf{R})=\left|\sigma_{g} \bar{\sigma}_{g} \sigma_{u}\right|,
$$

which, in a spin-free formalism, can be factorized into

$$
\Psi_{\mathrm{RHF}}(\mathbf{R})=\left(\sigma_{g}(1) \sigma_{u}(3)-\sigma_{g}(3) \sigma_{u}(1)\right) \sigma_{g}(2) .
$$

It is appropriate to note that this approximate wave function does not belong to the same symmetry group as the exact wave function, but rather to a higher symmetry group. It is easy to see this fact by noticing that each orbital, being of $\sigma$ type, is a function of only two coordinates, $r_{A}$ and $r_{B}$ for example; only six variables are then needed to specify the RHF wave function. The higher symmetry is apparent if, for example, we consider the rotation of a single electron around the $z$ axis, keeping the other two electrons fixed. The RHF wave function is invariant with respect to this rotation, but the exact wave function is not. A different way to say the same thing is that the RHF wave function does not depend, neither explicitly nor implicitly, on the interparticle coordinates.

Let us now consider the node of this approximate wave function. Since $\Psi_{\mathrm{RHF}}$ is described using only six coordinates, we can loosely say that the node is a five-dimensional object, but due to the factorization of $\Psi_{\mathrm{RHF}}$ into $\alpha$ and $\beta$ determinants there is a further reduction of the dimensionality of the node, which can be described with only three coordinates. The RHF node is completely independent from the distance between the two $\alpha$ electrons and from the position of the $\beta$ electron. The antisymmetric part of the RHF wave function

$$
\sigma_{g}(1) \sigma_{u}(3)-\sigma_{g}(3) \sigma_{u}(1)
$$

does not define the node structure by geometric symmetry. The wave function changes sign upon inversion through the center of the molecular ion, but, because an infinite number of $\sigma_{g}$ and $\sigma_{u}$ orbitals can be used, the node structures of the resulting RHF wave functions are different.

It is worth to remind that the nodes of a many-body wave function are not directly related to the nodes of the single-particle orbitals. In $\mathrm{He}_{2}^{+}$the lowest $\sigma_{g}$ orbital has no nodes, while the first $\sigma_{u}$ orbital, by symmetry arguments, has 
TABLE I. $\mathrm{He}_{2}^{+}$: basis sets and fixed-node energies in hartrees.

\begin{tabular}{|c|c|c|c|c|c|}
\hline Basis set & & $\begin{array}{c}\text { RHF } \\
\text { orbitals }\end{array}$ & $\begin{array}{c}\text { CAS } \\
\text { orbitals }\end{array}$ & $\begin{array}{c}\text { CAS-NO } \\
\text { orbitals }\end{array}$ & $\begin{array}{l}\text { CI-NO } \\
\text { orbitals }\end{array}$ \\
\hline A & $1 s$ & $-4.9905(2)$ & & & \\
\hline B & $2(1 s)$ & $-4.9913(2)$ & & & \\
\hline $\mathrm{C}$ & $1 s 2 s$ & $-4.9938(2)$ & $-4.9444(5)$ & $-4.8499(5)$ & $-4.8503(6)$ \\
\hline $\mathrm{D}$ & $4(1 s)$ & $-4.9940(2)$ & & & \\
\hline$E^{a}$ & $5(1 s)$ & $-4.9926(2)$ & $-4.9939(2)$ & $-4.9921(2)$ & $-4.9918(2)$ \\
\hline $\mathrm{F}$ & $2(1 s) 2 s 3 s$ & $-4.9943(2)$ & $-4.9925(1)$ & $-4.9916(2)$ & $-4.9917(2)$ \\
\hline G & $1 s 2 p$ & $-4.9938(1)$ & $-4.9935(1)$ & $-4.9932(1)$ & $-4.9936(1)$ \\
\hline $\mathrm{H}$ & $2(1 s) 2 s 3 s 2 p$ & $-4.9939(1)$ & $-4.9941(1)$ & $-4.9936(1)$ & $-4.9936(1)$ \\
\hline $\mathrm{I}^{\mathrm{b}}$ & $2(1 s) 2 s 3 s 2(2 p)$ & $-4.9932(2)$ & & & \\
\hline Exact energy & & $-4.9945^{\mathrm{c}}$ & & & \\
\hline
\end{tabular}

the $x y$ plane as a node. While the nodes of the many-body approximate wave functions are in general dependent on the basis set expansion, the $\sigma_{u}$ orbital node does not change.

\section{Atomic-orbital basis set effect}

We begin analyzing the effect of the atomic basis set on the quality of the nodal surfaces within the framework of the RHF method. We selected the basis set optimized for $\mathrm{He}_{2}^{+}$by Reagan et al. $^{24}$ and the atomic Hartree-Fock basis set by Clementi and Roetti ${ }^{21}$ and examined the effect of the various atomic orbitals on the nodal surfaces. For each set the RHF wave function was computed and its energy and the corresponding FN-DMC energy are reported in Table I.

All calculations were performed at the equilibrium distance of 2.0626 bohrs. $^{25}$ We estimated a nonrelativistic limit of -4.9945 hartree from the values computed by Cencek and Rychlewski. $^{26}$

The first consideration that can be made is that a singledeterminant wave function built with only $s$ orbitals (basis F) is able to correctly predict the energy, at least to chemical accuracy. This fact is quite surprising in the light of the symmetry considerations made in the previous section, as the number of coordinates on which the exact nodal surface depends is drastically reduced from eight to three on going from the exact to the HF wave function. Since a nodal surface is defined implicitly by an equation which, in this case, cannot be solved analytically, it is not possible to look at the analytical structure of the node. However, since the RHF wave function does not depend on the interparticle distances, the same applies to the RHF node; but since the RHF fixednode energy is, within chemical accuracy, equal to the exact energy, we can infer that the exact nodal surface is independent or perhaps very weakly dependent from the interparticle coordinates. Furthermore, we have previously seen that the nodal surface of the RHF wave function is also independent from the coordinates of the $\beta$ electron, so we can conclude again that the exact nodal surface is independent, or perhaps very weakly dependent, on the coordinates of the $\beta$ electron. A more mathematically precise statement is that the nodal surface belongs to a higher-symmetry group than the wave function itself. Far from being a mathematical curiosity, this fact has important practical consequences, as we will see.

The unexpected higher symmetry of the nodal surfaces for many-body systems has been previously noted for the helium case, ${ }^{16,27}$ where in some states it is possible to prove it analytically, and numerically for the lithium atom ground state. ${ }^{16}$ Also the exact nodes of spin-polarized states of few two- and three-electron systems show a higher symmetry than would be expected solely from antisymmetry. ${ }^{28}$ Apparently the $\mathrm{He}_{2}^{+}$ground state is yet another example of this, still theoretically unexplained, phenomenon. The first, and most important consequence, is that two somewhat contrasting factors are at play: the exact wave function depends on the interparticle distances and on the coordinates of the $\beta$ electron, and so to converge toward the exact wave function one must include all the relevant coordinates. At the same time, however, convergence to the exact wave function nodes can be partially disrupted, since apparently the nodes depend on fewer coordinates, and there must be a very delicate cancellation of terms right at the node in order to converge towards the exact node.

This observation might explain some of the seemingly bizarre behavior that has been sometimes reported in the literature, ${ }^{13}$ where, upon the improvement of a trial wave function by using a larger basis, the quality of the related nodal surface is unchanged or even decreased. The energy for basis $\mathrm{H}$, obtained by adding a $p$ atomic orbital to basis $\mathrm{F}$, is higher, so the extra functions have spoiled the nodal surface.

Usually it is not possible to have a visual representation of the nodal surfaces due to their high dimensionality. However, in this case we can take advantage of the fact that the nodes of RHF wave functions depend only on the coordinates of the two $\alpha$ electrons. To further reduce the dimensionality of the plots we decided to fix the position of the first $\alpha$ electron along the $z$ axis. Taking advantage of the cylindrical symmetry of the system, we constrained the second $\alpha$ electron to the $y z$ plane, so that we can make a contour plot by joining all the coordinates of the second electron, such that the wave function is zero. Cuts of the nodal surfaces for four basis sets are plotted in Fig. 1. The black small 

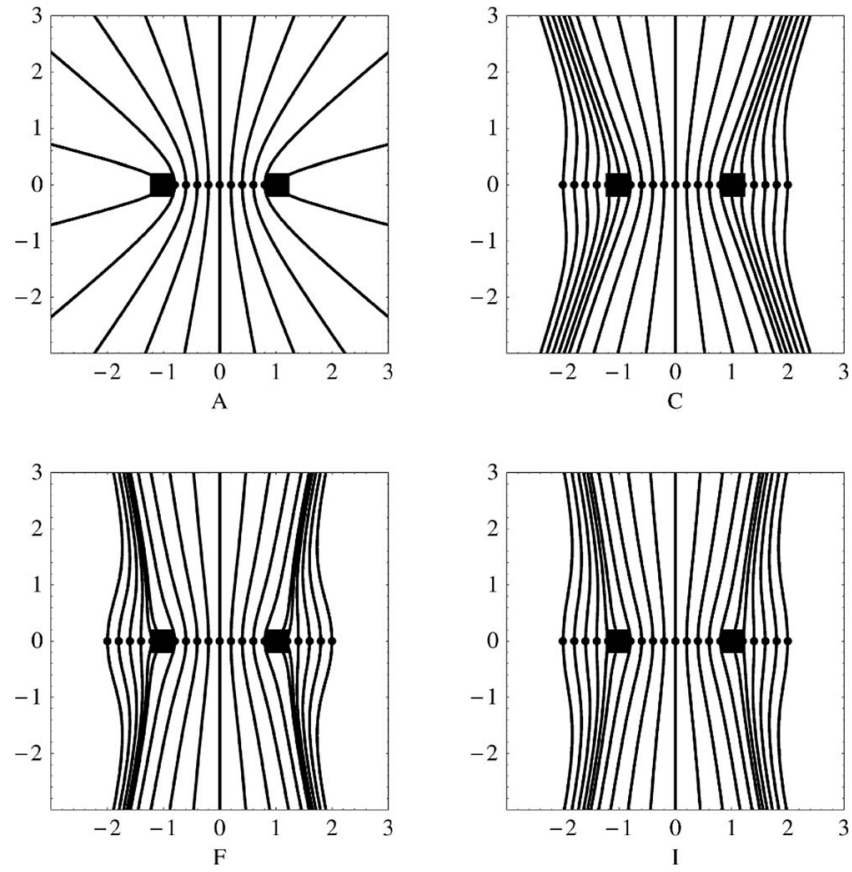

FIG. 1. $\mathrm{He}_{2}^{+}$: cuts of the nodal surfaces for RHF wave functions with basis sets defined in Table I.

dots represent the various positions where we fixed the first $\alpha$ electron, while the bigger squares represent the positions of the nuclei. All the curves pass through one black dot, since the wave function must be zero whenever two like electrons are at the same point. Examining the sign domains for a wider range of $y$ and $z$ coordinates, no nodal artifacts were observed as found in Be atom by Hachmann et al. ${ }^{12}$ So plots are reported in the range $[-3,+3]$ bohrs from the center of the molecular ion. The best nodal surfaces are those of the RHF wave function for basis F. All the surfaces are given approximately by $\psi=0$ for $z_{1}=z_{3}$, a situation similar to the one found in the triplet state ${ }^{3} \Sigma_{u}^{+}$of the $\mathrm{H}_{2}$ molecule. ${ }^{29}$

We can take advantage of the fact that cuts of the nodal surfaces can be visualized to get some insight when comparing different wave functions.

It is reasonably easy in FN-DMC simulations for atoms and molecules to recover $80 \%-90 \%$ of the correlation energy, using a moderately small basis set. However, there is not yet a consensus on which strategy is better if one wants to recover the remaining correlation energy, which appears to be a much more difficult task than originally thought. Enlarging the basis set, for a given trial wave function, is surely an option. However, it is far from clear which is the best way to proceed in order to improve the quality of the basis set. Let us now examine the effect of enlarging the atomic basis set on the nodal surfaces of our model system.

We start from the minimal basis set wave function (basis A). A curious feature of this wave function is that the nodal surface is independent from the exponent of the $1 \mathrm{~s}$ Slater orbital centered on the nuclei. A little algebra shows that the equation of the node is

$$
r_{1 A}-r_{1 B}=r_{3 A}-r_{3 B},
$$

which is a family of hyperboloid surfaces. Similarly to the previously seen case of the $\mathrm{Be}$ atom wave functions built with only $s$ atomic orbitals, this is another explicit proof that the variational energy of a trial wave function is not directly related to its fixed-node energy: different values of the exponent of the $1 s$ orbital would change the variational energy, but not the shape of the nodal surface.

Cuts of the nodal surfaces for basis A are shown in Fig. 1(A). While the DMC energy of this trial wave function is about 4 mhartrees away from the exact energy, nevertheless it is interesting that such a simple analytical form for the node can recover a substantial part of the correlation energy. In principle one can imagine a scheme for systematically improving the ansatz for the node itself, starting from this simple analytical form by including higher-order terms. However, we have not yet explored this possibility.

Comparing the nodes of Figs. 1(A) and 1(F) one can see that the nodes of the worse wave function show a higher curvature. This finding is consistent with the intuitive argument that nodes with a higher curvature increase the global curvature of the wave function, which in turn increases the local kinetic energy and the local energy.

To improve the quality of the minimal basis set A one could either add more $1 s$-type functions or introduce $2 s$ functions. Adding a second $1 s$ function (basis B) allows us to recover a FN-DMC energy of -4.9913(2) hartree, worse than $-4.9938(2)$ hartree of basis $\mathrm{C}$, built adding instead a $2 s$ function [see Fig. 1(C)]. We reoptimized the atomic-orbital exponents, but the fixed-node energy came out higher, so the effect of the atomic orbitals of the original basis sets was investigated without further optimization of the exponents. To recover an energy value equal in a statistical sense to the one of basis $\mathrm{C}$ using only $1 \mathrm{~s}$ orbitals, one has to include as many as four (basis D). In both cases there is a substantial gain in the energy with respect to the minimal basis set. The plots look similar to the plot of the exact node, and the energies are close to the exact value. However, the number of atomic orbitals doubles on going from the first function to the second one. It is clear that it is far more efficient to add functions with a higher principal quantum number than to use more functions of the same type. Consider now what happens if we add yet another $1 s$ function to basis set D. While the resulting wave function (basis $\mathrm{E}$, the atomic Hartree-Fock basis set by Clementi and Roetti ${ }^{21}$ ) has a lower RHF energy (-4.9082 against -4.9049 hartree), its fixednode energy is higher [-4.9926(2) against -4.9940 (2) hartree]. A plot of the nodal surface cuts shows an increased curvature.

This behavior, unfortunately, appears not to be uncommon to DMC simulations. It seems that, after a certain quality of the nodes has been achieved with a basis set, it is difficult to enlarge the basis without decreasing it. Apparently, too large basis sets do more harm than good. Unfortunately it is not yet possible to know how many functions of the same kind it is possible to add to the basis without spoiling the quality of the nodes.

A similar comparison can be performed by including, as customary in $a b$ initio calculations, polarization functions, that is, basis functions with a higher angular momentum. A simple basis composed of a single $1 s$ function and a single $2 p$ function (basis $\mathrm{G}$ ) gives a DMC energy very close to the 
exact energy [-4.9938(1) hartree], greatly improving the nodes, in comparison to the nodes of the minimal basis set wave function. This result is similar to the one computed adding a $2 s$ atomic orbital. However, adding a $p$ function (see basis $\mathrm{H}$ ) to the best basis composed by $s$ functions (basis F), the RHF energy improves, as it should ( -4.9219 against -4.9102 hartree), but the fixed-node energy increases [-4.9932(2) hartree], being slightly worse than the one of the simple $1 s-2 p$ wave function. Further addition of polarization functions of the basis set by Reagan et al. ${ }^{24}$ progressively spoils the nodal surfaces, even if the effect is tiny, as shown in Fig. 1(I).

We also tried to reoptimize all the parameters of the total wave function, either by minimizing the variance or the absolute deviation of the local energy: all the attempts resulted in a wave function with worse fixed-node energy.

From the above discussion we can draw some tentative conclusions: first of all, to enlarge a basis it seems more efficient to add functions with a higher principal quantum number rather than to add more functions of the same type. A second lesson to be learned is that apparently it is not possible to arbitrarily enlarge a basis set: although the variational energy improves, after a certain size of the basis the quality of the nodes can decrease. Unfortunately it is not possible to know in advance when the quality of the nodes will start to degrade.

\section{Single-particle basis set effect}

The second effect we want to investigate is the influence of the different single-particle orbitals that can be used in the wave-function construction.

Beyond HF orbitals, multiconfiguration-self-consistentfield- (MCSCF) generated orbitals ${ }^{15,30}$ and natural orbitals ${ }^{14}$ have been employed with success. More recently pair natural orbitals have been used to study the nitrogen and the water molecules. ${ }^{31}$ Also DFT methods have been used to generate orbitals. ${ }^{13,32}$ Usually it has been implicitly assumed that the nodes of a RHF wave function would give the best possible nodes, within a single-determinant description. In this section we investigate if this is true.

For a few selected atomic-orbital basis sets we performed four simulations using monodeterminantal wave functions built using RHF orbitals, optimized CAS orbitals, and natural orbitals computed from CAS and CI calculations. The resulting FN-DMC energies are shown in Table I.

The results differ significantly, which means that the nodal surfaces are somewhat different. Rather surprisingly, the RHF wave function is not always the best singledeterminant wave function, with respect to the fixed-node energy for all considered bases. CAS orbitals give the best fixed-node energy with bases $\mathrm{E}$ and $\mathrm{H}$, while RHF orbitals perform better with bases $\mathrm{C}, \mathrm{F}$, and $\mathrm{G}$. It is interesting to note that CAS orbitals perform better for the basis sets that are too large at the RHF level, those for which the inclusion of extra atomic orbitals resulted in spoiling the nodal surfaces of the smaller basis set. In one case (basis C) CAS orbitals perform significantly worse. Natural orbitals, both CAS-NO and CI-NO, give almost always the worst result. A plot of the
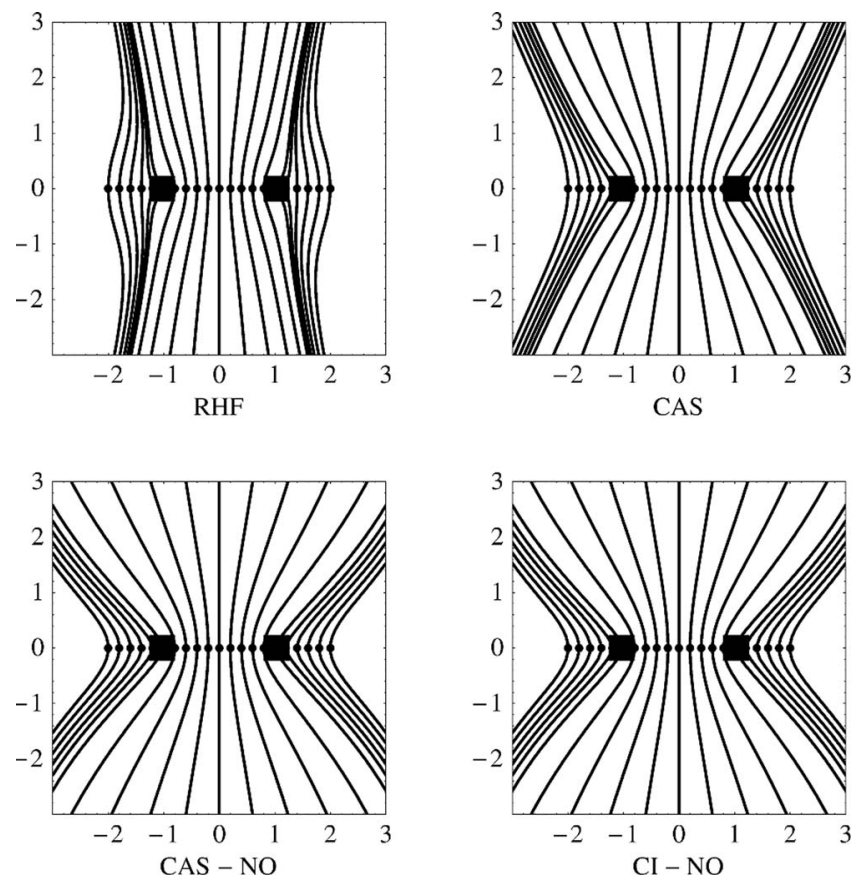

FIG. 2. $\mathrm{He}_{2}^{+}$: cuts of the nodal surfaces for monodeterminantal wave functions built with different single-particle orbitals (basis F).

various nodal surfaces, shown in Fig. 2, evidences that the curvature of the nodal surfaces increases on going from RHF to CAS to NO orbitals. Also DFT orbitals, generated using several different functionals, have been tested, but the results were worse and are not reported in Table I.

\section{Approximate wave functions: Multideterminant expansion}

Although in early quantum Monte Carlo (QMC) calculations the trial wave function used was typically of HF type, in recent years it has become common to employ multideterminant expansions, either from $\mathrm{MCSCF},{ }^{15}$ or $\mathrm{CAS}^{30}$ calculations, sometimes with hundreds of terms, ${ }^{13,31}$ with a high computational cost. While it is obvious that the variational energy improves with the length of the determinantal expansion, there are cases, reported in the literature, ${ }^{13,31}$ where, upon increasing the length of the configurations expansion, the fixed-node energy does not change, or even worse, increases. No explanation has been put forward for this phenomenon, and unfortunately so far there appears to be no general criterion to choose configurations to be included into the expansion with the aim to lower the fixed-node energy. While it appears reasonable, even if not mathematically proven, that as the variational energy of the expansion converges to the exact energy the nodes of the trial wave function converge to the exact nodes, the convergence of the fixed-node energy to the exact energy does not need to be monotonous, and indeed sometimes it is not. Sometimes the fixed-node energy is unchanged upon the addition of several determinants, in other cases the addition of a single determinant to the expansion can spoil the nodes of the trial wave function, and several more determinants might be needed to counterbalance this effect, with a substantial computational cost. It would be desirable to have a procedure to select in 
TABLE II. $\mathrm{He}_{2}^{+}$: multideterminant expansions. Energies in hartrees.

\begin{tabular}{|c|c|c|c|c|c|}
\hline $\begin{array}{l}\text { Basis } \\
\text { set }\end{array}$ & $\begin{array}{c}\text { Energy } \\
\text { single determinant }\end{array}$ & Added CSF's & $\begin{array}{c}\text { Energy } \\
\text { (RHF orbitals) }\end{array}$ & $\begin{array}{c}\text { Energy } \\
\text { (CAS orbitals) }\end{array}$ & $\begin{array}{c}\text { Energy } \\
\text { extra CSF's } \\
\text { (RHF orbitals) }\end{array}$ \\
\hline $\mathrm{C}$ & $-4.9938(2)$ & $1 \sigma_{g}^{1} 1 \sigma_{u}^{1} 2 \sigma_{g}^{1}$ & $-4.9778(3)$ & $-4.9753(1)$ & $-4.9503(3)$ \\
\hline $\mathrm{F}$ & $-4.9943(2)$ & $1 \sigma_{g}^{1} 1 \sigma_{u}^{1} 2 \sigma_{g}^{1}$ & $-4.9925(1)$ & $-4.9908(1)$ & $-4.9924(1)$ \\
\hline G & $-4.9938(1)$ & $1 \sigma_{u}^{1} 1 \pi_{u x}^{2}+1 \sigma_{u}^{1} 1 \pi_{u y}^{2}$ & $-4.9928(1)$ & $-4.9926(1)$ & $-4.9929(1)$ \\
\hline $\mathrm{H}$ & $-4.9932(2)$ & $1 \sigma_{u}^{1} 1 \pi_{u x}^{2}+1 \sigma_{u}^{1} 1 \pi_{u y}^{2}$ & $-4.9946(2)$ & $-4.9939(1)$ & $-4.9933(1)$ \\
\hline
\end{tabular}

advance only those terms in a CI expansion contributing to lower the fixed-node energy. This would ensure a more reliable DMC simulation and a reduced computational cost.

In order to investigate this matter, we performed a few tests, using different multideterminant expansions. The results are reported in Table II.

Adding the excited CI configuration with the largest coefficient, namely, $1 \sigma_{g}^{1} 1 \sigma_{u}^{1} 2 \sigma_{g}^{1}$, to the RHF (basis C) wave function, the fixed-node energy worsens dramatically, going from $-4.9938(2)$ to $-4.9778(3)$ hartree. The energy is even worse if CAS orbitals are used in this two-determinant expansion, and this is true for all basis sets considered in Table II. The same increase of the fixed-node energy happens also with basis F, even if the effect is reduced. The opposite improvement of the quality of the nodes is observed by adding the first excited CI configuration $1 \sigma_{u}^{1} 1 \pi_{u x}^{2}+1 \sigma_{u}^{1} 1 \pi_{u y}^{2}$ for basis $\mathrm{H}$; the resulting fixed-node energy is $-4.9946(2)$ hartree, which is the exact energy within the statistical error.

However, if we build a two-configuration wave function using basis $\mathrm{G}$, which performs better than basis $\mathrm{H}$ at the RHF FN-DMC level, it is interesting to note that the resulting energy slightly increases. It is more difficult to improve a good trial wave function by adding more configurations than to improve a relatively bad wave function. However, we note that the configurations that can be added to the singledeterminant wave functions built using bases $\mathrm{C}$ and $\mathrm{F}$ involve only $\sigma$ orbitals. It seems that the addition of configurations, built using orbitals with the same angular momentum of those used to build the single determinant, spoils the quality of the nodes. Basis sets $\mathrm{G}$ and $\mathrm{H}$ instead generate orbitals with a higher angular momentum, and basis $\mathrm{H}$, with two configurations, is able to correctly describe the exact nodes. Including more configurations either leaves the fixed-node energy unchanged or leads to its increase.

It is interesting to compare the performance of the two very small basis sets $\mathrm{C}(1 s 2 s)$ and $\mathrm{G}(1 s 2 p)$. They give the same FN energy when used to build a single-determinant wave function, and better than basis B (see Table I). However, they behave differently when used to build a multideterminant expansion. While in the case of basis $\mathrm{C}$ there is a complete disruption of the nodes, using basis $\mathrm{G}$ leads only to a small perturbation of the nodes. It is clear that, while in both cases they provide an adequate description of the single-determinant wave function, there is not enough flexibility in basis $\mathrm{G}$ to accurately describe the $1 \sigma_{u}^{1} 1 \pi_{u x}^{2}$ $+1 \sigma_{u}^{1} 1 \pi_{u y}^{2}$ configuration, which appears to be able to correct the inaccuracies of the node of the single determinant when using basis $\mathrm{H}$. It is worth to remind that the excited configu- rations $1 \sigma_{g}^{1} 1 \sigma_{u}^{1} 2 \sigma_{g}^{1}$ and $1 \sigma_{u}^{1} 1 \pi_{u x}^{2}+1 \sigma_{u}^{1} 1 \pi_{u y}^{2}$ belong to different symmetry species of a higher-symmetry group of the Hamiltonian. These symmetry species are those of the model Hamiltonian with all electron-electron interactions turned off.

A few tentative conjectures can be drawn from all these numerical experiments: the basis set should be fairly large to recover as much correlation energy as possible. Given that we are studying a three-electron system, the basis needed to recover the exact energy seems to be larger than previously thought. It is still common to employ, in the DMC literature, double-zeta quality basis sets.

Our findings seem to show that larger basis sets should be used. However, care must be taken when choosing the basis functions: it appears more useful to include functions with a higher principal quantum number than functions with the same principal quantum number and a different exponent. When building a multideterminant expansion it seems that including configurations built on orbitals of the same symmetry as those of the ground state leads to a decrease of the quality of the nodes. In this light higher angular momentum functions should be included in the basis set. Basis F, while building a very good single-determinant node, cannot generate configurations of different symmetries. Basis $\mathrm{H}$ on the other hand, while not as good as basis F at the singledeterminant level, is able to generate a configuration of the needed symmetry, and gives the exact energy. Considering different ways to generate the orbitals to be included into multideterminant wave functions, it seems that the optimal strategy depends on the quality of the basis set. For good atomic-orbital basis sets RHF orbitals used in a CI expansion give the best results. With lower-quality basis sets, CAS seems to perform better. Natural orbitals are the last choice.

\section{$\mathrm{Li}_{2}$ MOLECULE}

The system we studied next is the $\mathrm{Li}_{2}$ molecule in its ground state, at an equilibrium distance of 5.051 bohrs. ${ }^{33} \mathrm{We}$ chose this system because it is nontrivial and there are a few published QMC calculations ${ }^{17,31,34-38}$ that we can compare with.

It was not possible to perform a systematic study for $\mathrm{Li}_{2}$ as we did for $\mathrm{He}_{2}^{+}$, but we tried to see if the empirical rules that we found for $\mathrm{He}_{2}^{+}$were valid also for this bigger system.

Single-determinant FN-DMC calculations have been reported in the literature: Reynolds et $a .^{34}$ used double-zeta basis sets; their best result was -14.991(7) hartree. Huang and $\mathrm{Liu}^{38}$ at the zeroth order of their eigenvalue expansion 
TABLE III. $\mathrm{Li}_{2}$ DMC energies in hartrees for multideterminant trial wave functions. We omit the core double occupied molecular orbitals $1 \sigma_{g}^{2} 1 \sigma_{u}^{2}$.

\begin{tabular}{lccl}
\hline \hline CSF & Ndet & Additional CSF & DMC energy \\
\hline 1 & 1 & $2 \sigma_{g}^{2}$ & $-14.9923(2)$ \\
8 & 8 & $3 \sigma_{g}^{2}+4 \sigma_{g}^{2}+\cdots+9 \sigma_{g}^{2}$ & $-14.9914(2)$ \\
2 & 3 & $1 \pi_{u x}^{2}+1 \pi_{u y}^{2}$ & $-14.9933(2)$ \\
5 & 9 & $1 \pi_{u x}^{2}+1 \pi_{u y}^{2}+\cdots+4 \pi_{u x}^{2}+4 \pi_{u y}^{2}$ & $-14.9933(1)$ \\
3 & 4 & $1 \pi_{u x}^{2}+1 \pi_{u y}^{2}+2 \sigma_{u}^{2}$ & $-14.9939(2)$ \\
4 & 5 & $1 \pi_{u x}^{2}+1 \pi_{u y}^{2}+2 \sigma_{u}^{2}+3 \sigma_{g}^{2}$ & $-14.9952(1)$ \\
Exact energy & & & $-14.9954^{\mathrm{a}}$ \\
\hline \hline
\end{tabular}

${ }^{\mathrm{a}}$ Reference 37.

method computed a value of $-14.9818(4)$ hartree with a minimal basis set. A value of -14.9890(2) hartree was computed by Umrigar et al. ${ }^{17}$ with a $2(1 s) 2 s 2 p$ basis set. Filippi and Umrigar ${ }^{37}$ with a double-zeta plus polarization basis set obtained -14.9911(1) hartree, while a value of $-14.9919(6)$ hartree was computed by Luchow and Fink ${ }^{31}$ with a larger basis. These results suggest that, as already found in the study on $\mathrm{He}_{2}^{+}$, a double-zeta quality basis set is insufficient to reach convergence of the HF nodes. For this reason we tested several larger basis sets taken from the literature, namely, a $6 s 4 p 2 d 1 f$ basis set, ${ }^{39}$ the atomic HF basis set, $^{21}$ and a basis set optimized for the dimer by Cade and Wahl. ${ }^{33}$ FN-DMC calculations were performed using a single determinant, built with single-particle HF or CAS orbitals, as explained in the previous section. The results are consistently better than the literature values computed with smaller basis sets.

The best FN-DMC energy, -14.9923(2) hartree, was obtained using the basis set by Liu et al. ${ }^{39}$ without $d$ and $f$ orbitals (basis A in the following), as they spoil the nodal surfaces. Since the energy is far from the nonrelativistic limit -14.9954 hartree, the single-determinant nodes have not yet reached convergence. As previously found for $\mathrm{He}_{2}^{+}$, in this situation CAS single-particle orbitals perform better than $\mathrm{HF}$ orbitals.

It is interesting to note that our single-determinant wave function recovers as much correlation energy as a fourdeterminant wave function built with a smaller basis. ${ }^{37}$ This confirms our tentative conjecture that before starting to add different configurations, if it is computationally feasible, it is a good idea to check whether the single-determinant nodes have reached convergence, using a sufficiently large basis set. Sometimes configurations that seemed important in order to improve the energy came out to be useless if a larger basis was used. Unfortunately there appears to be yet no way to know beforehand if an employed basis set is sufficiently large.

Unlike the $\mathrm{He}_{2}^{+}$case, our best single-determinant wave function does not reach the chemical accuracy, and the use of a multideterminant expansion is necessary to converge to the exact energy.

We now proceed to add configurations using CAS orbitals of basis A that performed better at the single-determinant level: the results are shown in Table III.

Adding to the ground-state configuration up to seven CSFs of the type $2 \sigma_{g}^{2} \rightarrow n \sigma_{g}^{2}$ results in a sensible increase of
TABLE IV. $\mathrm{C}_{2}$ DMC energies in hartrees for a single-determinant trial wave function.

\begin{tabular}{cccc}
\hline \hline & $\mathrm{A}^{\mathrm{a}}$ & $\mathrm{B}^{\mathrm{b}}$ & $\mathrm{C}^{\mathrm{c}}$ \\
\hline CAS & $-75.8638(6)$ & $-75.8591(6)$ & $-75.8612(3)$ \\
NO & $-75.8594(4)$ & $-75.8642(5)$ & $-75.8694(3)$ \\
\hline
\end{tabular}

${ }^{\mathrm{a}}$ Basis set from Ref. 33 .

${ }^{\mathrm{b}}$ Basis set from Ref. 21.

${ }^{c}$ Basis set from Ref. 30.

the energy, the same is found using $\sigma_{u}$ orbitals instead of $\sigma_{g}$. As already noted in the $\mathrm{He}_{2}^{+}$case, the nodes are spoiled by excitations to orbitals with the same angular momentum as those occupied at the HF level. To improve the energy one has to include excitations to orbitals with a higher component of the angular momentum along the internuclear axis, in this system double excitations to $\pi$ orbitals. Neither adding further configurations of the same kind, up to four, nor excitations to orbitals of an even higher angular momentum, namely, $\delta$ orbitals, changes the energy. The next most important configuration to add to the three-determinant wave function is the double excitation to a $\sigma_{u}$ orbital with a strong $p_{z}$ character; the excitation instead to a $\sigma_{g}$ orbital gives a smaller improvement. However, its contribution becomes relevant after the inclusion of the excitation $\sigma_{g}^{2} \rightarrow \sigma_{u}^{2}$ and this five-configuration wave function allows us to recover, within two standard deviations, the exact energy. So excitations to orbitals with the same angular momentum might be effective only after orbitals with a higher angular momentum have contributed to improve the wave function. An alternative conjecture is that the contribution from the $\sigma_{g}^{2} \rightarrow \sigma_{g}^{2}$ excitation would disappear by using an even larger basis, as observed in the previously presented cases. In conclusion the relevant configurations are those describing angular, radial, and left-right correlation. The same trend can be evidenced from the results by Filippi and Umrigar, ${ }^{37}$ even if their inclusion of the excitation to the $\sigma_{u}$ orbital before the excitation to the $\pi_{u}$ orbital apparently implies a negative effect of the corresponding configuration. In conclusion only five determinants are required to build a nearly exact nodal surface. Our energy is $-14.9952(1)$ hartree; the best previous QMC result, obtained by Luchow and Fink, ${ }^{31}$ was $-14.9948(1)$ hartree.

\section{$\mathrm{C}_{2}$ MOLECULE}

$\mathrm{C}_{2}$ is a challenging system to computational chemistry owing to its multireference nature that presents problems to single-reference methods. For example, $\operatorname{CCSD}(\mathrm{T})$ calculations with large basis sets cannot achieve chemical accuracy. ${ }^{40}$ Filippi and Umrigar ${ }^{37}$ outlined the difficulty in the treatment of this system with quantum Monte Carlo: comparing DMC results of the first-row diatomic molecules, at a multideterminant level $\mathrm{C}_{2}$ provides the worst energy result, recovering less correlation energy percentage than the other first-row diatomic molecules. Barnett et al. $^{30}$ showed that DMC can recover more than $96 \%$ of the correlation energy, if one uses a large multideterminant expansion and a large basis set. This was also found by Luchow and Fink, ${ }^{31}$ 
TABLE V. $\mathrm{C}_{2}$ DMC energies in hartrees for a multideterminant trial wave function built with NO single-particle orbitals and basis $\mathrm{C}$. We omit the core double occupied molecular orbitals $1 \sigma_{g}^{2} 1 \sigma_{u}^{2}$.

\begin{tabular}{lccc}
\hline \hline CSF & Ndet & Additional CSF & DMC energy \\
\hline 1 & 1 & $2 \sigma_{g}^{2} 1 \pi_{u}^{x^{2}} 1 \pi_{u}^{y^{2}} 2 \sigma_{u}^{2}$ & $-75.8694(3)$ \\
2 & 2 & $2 \sigma_{g}^{2} 1 \pi_{u}^{x^{2}} 1 \pi_{u}^{y^{2}} 3 \sigma_{g}^{2}$ & $-75.8859(3)$ \\
3 & 10 & $2 \sigma_{g}^{2} 1 \pi_{u}^{x^{2}} 1 \pi_{u}^{y^{1}} 2 \sigma_{u}^{1} 3 \sigma_{g}^{1} 1 \pi_{g}^{y^{1}}$ & $-75.8944(4)$ \\
4 & 12 & $2 \sigma_{g}^{2} 1 \pi_{u}^{x^{2}} 2 \sigma_{u}^{2} 1 \pi_{g}^{y^{2}}$ & $-75.8962(3)$ \\
6 & 22 & $2 \sigma_{g}^{2} 1 \pi_{u}^{x^{1}} 1 \pi_{u}^{y^{1}} 2 \sigma_{u}^{2} 1 \pi_{g}^{x^{1}} 1 \pi_{g}^{y^{1}}$ & $-75.8945(6)$ \\
7 & 24 & $2 \sigma_{g}^{2} 1 \pi_{u}^{x^{2}} 1 \pi_{u}^{y^{2}} 1 \pi_{g}^{x^{2}}$ & $-75.8997(4)$ \\
8 & 32 & $2 \sigma_{g}^{1} 1 \pi_{u}^{x^{1}} 1 \pi_{u}^{y^{2}} 2 \sigma_{u}^{2} 1 \pi_{g}^{x^{1}} 3 \sigma_{u}^{1}$ & $-75.9032(8)$ \\
$8^{\mathrm{a}}$ & 32 & $2 \sigma_{g}^{1} 1 \pi_{u}^{x^{1}} 1 \pi_{u}^{y^{2}} 2 \sigma_{u}^{2} 1 \pi_{g}^{x^{1}} 3 \sigma_{u}^{1}$ & $-75.9038(6)$ \\
4 & 16 & & $-75.8901(7)^{\mathrm{b}}$ \\
36 & & & $-75.9025(7)^{\mathrm{c}}$ \\
Exact energy & & & $-75.900(1)^{\mathrm{d}}$ \\
\hline \hline
\end{tabular}

${ }^{\mathrm{a}}$ Linear coefficients optimized at the DMC level.

${ }^{\mathrm{b}}$ Reference 37.

${ }^{c}$ Reference 30.

${ }^{\mathrm{d}}$ Reference 31.

who obtained very accurate results for $\mathrm{C}_{2}$ using a quite large MCSCF expansion built with the Cade-Wahl basis set. ${ }^{33}$ Overall, those results support our hypothesis that basis sets larger than previously thought must be used to describe the nodes adequately.

We first tested several basis sets taken from the literature and performed DMC simulations, at the experimental bond length of 2.3481 bohrs, ${ }^{33}$ using a single-determinant wave function. CAS and $\mathrm{NO}$ orbitals give better FN-DMC energies than HF orbitals: the results are shown in Table IV. To expand the wave function with excited configurations we decided to use NO orbitals and basis $\mathrm{C}$ that give the best result at the single-determinant level. Furthermore, its use allows us to perform a systematic comparison between our results and the ones reported by Barnett et al. ${ }^{30}$ FN-DMC energy results are reported in Table V.

We added up to seven CSFs to the ground state. Each inclusion improves the nodes, except for the $1 \pi_{u}^{x} 1 \pi_{u}^{y}$ $\rightarrow 1 \pi_{g}^{x^{1}} 1 \pi_{g}^{y^{1}}$ excitation that slightly raises the energy, but its inclusion is required together with the successive CSFs in
Table $\mathrm{V}$ to get the final value of $-75.9032(8)$ hartree, at present the best DMC value. We tried to add more CSFs, but we were not able to recover more correlation energy. On the contrary, an expansion of 23 configurations gave a worse energy [-75.8999(4) hartree]. With the same basis set and an optimized wave function containing $36 \mathrm{CFSs}$, Barnett et al. ${ }^{30}$ obtained $-75.9025(7)$ hartree. Our better result once again suggests that, when a good description of nodes is reached, the inclusion in the wave function of further CFSs spoils the nodal surface. Examining the configurations that contribute to improve the nodes, we observe that the first six involve excitations from the three highest doubly occupied orbitals and only the last CFS includes the excitation of a lower energy $\sigma$ orbital. Furthermore all CSFs involve excitations from $g$ to $u$ or from $u$ to $g$ orbitals, including so the left-right correlation. They correlate $\sigma$ and $\pi$ electron pairs and crosscorrelate $\sigma$ and $\pi$ electrons and $\pi$ electrons in different orbitals.

Optimization of the coefficients of the determinant expansion and of the molecular orbitals is another common way to improve the wave function, but it is computationally expensive. We tried to optimize the linear coefficients of the eight CSF expansions obtaining a value $-75.9038(6)$ hartree, recovering $96.3 \%$ of the correlation energy.

In conclusion, since in some cases FN-DMC energies became even worse after adding additional CSFs, it is important to select only configurations that improve the nodes: this selection allows not only a better description of the nodal surface, avoiding to spoil correct nodes, but also an appreciable reduction in the computational cost of the simulation.

\section{MORE DIMERS}

We applied the empirical rules found examining the previously discussed systems to other dimers of the first row. We do not present their results with the level of details already used; we limit ourselves to report the best FN-DMC energies in Table VI. The basis sets by Cade-Huo, ${ }^{41}$ optimized for the first-row hydrides, were selected instead of the ones optimized for first-row dimers ${ }^{33}$ as they are larger and so give better nodal surfaces. However, for $\mathrm{Be}_{2}$ we neglected the $d$ orbitals, as we did for $\mathrm{Li}_{2}$, and added an extra $2 p$

TABLE VI. DMC energies in hartrees for dimers.

\begin{tabular}{lcccccc}
\hline \hline Molecule & $\mathrm{CSF}^{\mathrm{a}}$ & $E_{\mathrm{DMC}}{ }^{\mathrm{a}}$ & $\mathrm{CSF}^{\mathrm{b}}$ & $E_{\mathrm{DMC}}{ }^{\mathrm{b}}$ & $\begin{array}{c}E_{\text {corr }} \\
(\%)\end{array}$ & $\begin{array}{c}\text { Exact }^{\mathrm{b}} \\
\text { energy }\end{array}$ \\
\hline $\mathrm{Be}_{2}$ & 1 & $-29.3178(2)$ & 1 & $-29.3176(2)$ & & $-29.33854(5)$ \\
& 4 & $-29.3336(2)$ & 5 & $-29.3301(2)$ & 95.9 & \\
$\mathrm{~B}_{2}$ & 1 & $-49.3813(1)$ & 1 & $-49.3778(8)$ & & $-49.415(2)$ \\
& 5 & $-49.3998(3)$ & 6 & $-49.3979(6)$ & 95.3 & \\
$\mathrm{~N}_{2}$ & 1 & $-109.5041(4)$ & 1 & $-109.487(1)$ & & -109.5423 \\
& 3 & $-109.512(1)$ & 4 & $-109.505(1)$ & 94.5 & \\
$\mathrm{O}_{2}$ & 1 & $-150.2850(6)$ & 1 & $-150.268(1)$ & & -150.3268 \\
& 2 & $-150.2869(6)$ & 4 & $-150.277(1)$ & 94.0 & \\
$\mathrm{~F}_{2}$ & 1 & $-199.4806(8)$ & 1 & $-199.478(2)$ & & -199.5299 \\
& 2 & $-199.4862(7)$ & & $-199.487(1)$ & 94.2 & \\
\hline \hline
\end{tabular}

\footnotetext{
${ }^{\mathrm{a}}$ Present work.
}

${ }^{\mathrm{b}}$ Reference 37. 
function, while for the other dimers the basis set was supplemented with an extra $d$ orbital. We did not check whether the nodal surfaces are at convergence with respect to the atomicorbital basis set. We compare our values with those by Filippi and Umrigar ${ }^{37}$ who made a systematic study of the firstrow dimers. For $\mathrm{Be}_{2}$ we get the same value with a singledeterminant wave function, but our value with four CSFs is lower than their -29.3301(2) hartree computed with five CSFs, the CSF associated with the second spin function for the excitation $2 \sigma_{g} 2 \sigma_{u} \rightarrow 1 \pi_{u} 1 \pi_{g}$ being detrimental to the FN-DMC energy. For $\mathrm{Be}_{2}$ the best FN-DMC energy of -29.3334(3) hartree was computed by Casula et al. $^{42}$ using a correlated geminal wave function; for the other systems examined in this work their approach gives worse energies. For $\mathrm{B}_{2}$ our better basis set allows us to improve over the values by Filippi and Umrigar both at the single-determinant level and when including five CSFs instead of their six CSFs. Again for the space configuration with four electrons in four orbitals it is enough to include only one spin function. The effect of a large basis set of atomic orbitals is even more evident in $\mathrm{N}_{2}, \mathrm{O}_{2}$, and $\mathrm{F}_{2}$. For $\mathrm{N}_{2}$ our best result was obtained using only three CSFs instead of their four CSFs, again including only one spin function for a given space configuration. A larger reduction of the expansion was found for $\mathrm{O}_{2}$ : only two CSFs are effective instead of their four CSFs, the excitations from $3 \sigma_{g}$ orbital being detrimental to the FN-DMC energy. For $\mathrm{F}_{2}$ we included the same CSFs, the better result depends on the larger atomic basis set.

As a general comment on the CSFs we found effective in improving the nodal surface, only excitations to valence bonding and the corresponding antibonding orbitals must be included in the CI expansion, the contribution of higherenergy orbitals is either negligible or harmful.

\section{CONCLUSIONS}

In this paper the problem of the so-called nodal error in FN-DMC simulations has been investigated in a series of atoms and molecules. The elements that influence the determinantal part of the wave function, namely, the atomic basis set, the single-particle orbitals, and the length of the determinantal expansion, were examined. A series of empirical rules can be extracted from this study. The atomic basis set must be larger than the double-zeta (DZ) basis sets and should be optimized on the molecule, ${ }^{33}$ not on the atoms. ${ }^{21}$ The cost of the optimization by ab initio methods is negligible with respect to the cost of the DMC simulation and does pay in reducing the nodal error and the energy variance. Usually CAS or NO single-particle orbitals are better than HF orbitals. Few configurations contribute to improve the nodal surface upon the single-determinant node; long expansions are not only expensive, but usually include CSFs that spoil the nodal surface. When more spin functions can be associated with a space configuration, in general only the CSF with the largest coefficient improves the nodal surface. Almost always, it is better to include CSFs built using orbitals with different orbital angular momenta.

Using these empirical findings, for a series of molecules we were able to compute FN-DMC energies that are better than almost all the previously published results. It might be possible to further improve these values, in particular, by enlarging the atomic basis set. However, even if we were able to reduce the nodal error, we do not recover much more than $95 \%$ of the correlation energy. Beyond a given point, enlarging the atomic basis set and including more CSFs do not improve the nodal surfaces. Our feeling is that the larger the number of electrons, the more difficult is to improve the nodal surfaces: for example, the number of CSFs that we found effective increases from four for $\mathrm{Li}_{2}$ to eight for $\mathrm{C}_{2}$ and then decreases to three for $\mathrm{N}_{2}$ and two for $\mathrm{O}_{2}$. The wave functions are very involved functions that result from the optimization of many linear and nonlinear parameters with respect to the energy. On the other hand we have seen that the nodal surfaces have simpler structures, so the more terms are present in the wave function, the more difficult is to define its zero by cancellation of terms of opposite sign.

\section{ACKNOWLEDGMENTS}

The authors would like to thank Carlos Bunge Molina for providing us various accurate CI wave functions for the Be atom and other atomic systems and for many discussions regarding atomic wave functions. This work was supported by Italian MIUR Grant No. 2004034838.

${ }^{1}$ J. B. Anderson, J. Chem. Phys. 63, 1499 (1975).

${ }^{2}$ D. M. Arnow, M. H. Kalos, M. A. Lee, and K. E. Schmidt, J. Chem. Phys. 77, 5562 (1982).

${ }^{3}$ S. Zhang and M. H. Kalos, Phys. Rev. Lett. 67, 3074 (1991).

${ }^{4}$ D. M. Ceperley and B. J. Alder, J. Chem. Phys. 81, 5833 (1984).

${ }^{5}$ M. Caffarel and P. Claverie, J. Chem. Phys. 88, 1100 (1988).

${ }^{6}$ J. B. Anderson, C. A. Traynor, and B. M. Boghosian, J. Chem. Phys. 95, 7418 (1991)

${ }^{7}$ M. H. Kalos and F. Pederiva, Physica A 279, 236 (2000).

${ }^{8}$ A. Luchow and J. B. Anderson, J. Chem. Phys. 105, 7573 (1996).

${ }^{9}$ G. Morosi, M. Mella, and D. Bressanini, J. Chem. Phys. 111, 6755 (1999).

${ }^{10}$ M. Casula and S. Sorella, J. Chem. Phys. 119, 6500 (2003).

${ }^{11}$ A. Luchow and J. B. Anderson, J. Chem. Phys. 105, 4636 (1996).

${ }^{12}$ J. Hachmann, P. T. A. Galek, T. Yanai, G. K. L. Chan, and N. C. Handy, Chem. Phys. Lett. 392, 55 (2004).

${ }^{13}$ J. C. Grossman, J. Chem. Phys. 117, 1434 (2002).

${ }^{14}$ J. C. Grossman and L. Mitas, Phys. Rev. Lett. 74, 1323 (1995).

${ }^{15}$ H. J. Flad, M. Caffarel, and A. Savin, in Recent Advances in Quantum Monte Carlo Methods, edited by W. A. Lester, Jr. (World Scientific, Singapore, 1997), Pt. I, p. 73.

${ }^{16}$ D. Bressanini, D. M. Ceperley, and P. J. Reynolds, in Recent Advances in Quantum Monte Carlo Methods, edited by W. A. Lester, Jr. (World Scientific, Singapore, 2002), Pt. II, p. 1.

${ }^{17}$ C. J. Umrigar, M. P. Nightingale, and K. J. Runge, J. Chem. Phys. 99, 2865 (1993)

${ }^{18}$ C. J. Huang, C. J. Umrigar, and M. P. Nightingale, J. Chem. Phys. 107, 3007 (1997).

${ }^{19}$ J. Komasa, J. Rychlewski, and K. Jankowski, Phys. Rev. A 65, 042507 (2002).

${ }^{20}$ C. F. Bunge (unpublished).

${ }^{21}$ E. Clementi and C. Roetti, At. Data Nucl. Data Tables 14, 177 (1974).

${ }^{22}$ D. Bressanini and P. J. Reynolds, Phys. Rev. Lett. 95, 110201 (2005).

${ }^{23}$ R. J. White and F. H. Stillinger, Jr., J. Chem. Phys. 52, 5800 (1970).

${ }^{24}$ P. N. Reagan, J. C. Browne, and F. A. Matsen, Phys. Rev. 132, 304 (1963).

${ }^{25}$ C. Edmiston and M. Krauss, J. Chem. Phys. 45, 1833 (1966).

${ }^{26}$ W. Cencek and J. Rychlewski, J. Chem. Phys. 102, 2533 (1995).

${ }^{27}$ J. B. Anderson, Phys. Rev. A 35, 3550 (1987).

${ }^{28}$ M. Bajdich, L. Mitas, G. Drobny, and L. K. Wagner, Phys. Rev. B 72, 075131 (2005). 
${ }^{29}$ J. B. Anderson, J. Chem. Phys. 65, 4121 (1976).

${ }^{30}$ R. N. Barnett, Z. W. Sun, and W. A. Lester, Jr., J. Chem. Phys. 114, 2013 (2001).

${ }^{31}$ A. Luchow and R. F. Fink, J. Chem. Phys. 113, 8457 (2000).

${ }^{32}$ L. Wagner and L. Mitas, Chem. Phys. Lett. 370, 412 (2003).

${ }^{33}$ P. E. Cade and A. C. Wahl, At. Data Nucl. Data Tables 13, 339 (1974)

${ }^{34}$ P. J. Reynolds, D. M. Ceperley, B. J. Alder, and W. A. Lester, Jr., J. Chem. Phys. 77, 5593 (1982).

${ }^{35}$ Z. W. Sun, S. Y. Huang, R. N. Barnett, and W. A. Lester, Jr., J. Chem. Phys. 93, 3326 (1990).
${ }^{36}$ Z. Sun, R. N. Barnett, and W. A. Lester, Jr., J. Chem. Phys. 96, 2422 (1992).

${ }^{37}$ C. Filippi and C. J. Umrigar, J. Chem. Phys. 105, 213 (1996).

${ }^{38}$ H. X. Huang and S. B. Liu, J. Mol. Struct.: THEOCHEM 636, 125 (2003).

${ }^{39}$ B. Liu, K. O-Ohata, and K. Kirby-Docken, J. Chem. Phys. 67, 1850 (1977).

${ }^{40}$ D. Feller and J. A. Sordo, J. Chem. Phys. 113, 485 (2000).

${ }^{41}$ P. E. Cade and W. Huo, At. Data Nucl. Data Tables 12, 415 (1973).

${ }^{42}$ M. Casula, C. Attaccalite, and S. Sorella, J. Chem. Phys. 121, 7110 (2004). 